\title{
Combining the bi-Yang-Baxter deformation, the Wess-Zumino term and TsT transformations in one integrable $\sigma$-model
}

\author{
F. Delduc, ${ }^{a}$ B. Hoare, ${ }^{b}$ T. Kameyama ${ }^{a}$ and M. Magro ${ }^{a}$ \\ ${ }^{a}$ Université Lyon, ENS de Lyon, Université Claude Bernard, CNRS, Laboratoire de Physique, \\ F-69342 Lyon, France \\ ${ }^{b}$ Institut für Theoretische Physik, Eidgenössische Technische Hochschule Zürich, \\ Wolfgang-Pauli-Strasse 27, 8093 Zürich, Switzerland \\ E-mail: francois.delduc@ens-lyon.fr, bhoare@itp.phys.ethz.ch, \\ takashi.kameyama@ens-lyon.fr, marc.magro@ens-lyon.fr
}

ABSTRACT: A multi-parameter integrable deformation of the principal chiral model is presented. The Yang-Baxter and bi-Yang-Baxter $\sigma$-models, the principal chiral model plus a Wess-Zumino term and the TsT transformation of the principal chiral model are all recovered when the appropriate deformation parameters vanish. When the Lie group is $\mathrm{SU}(2)$, we show that this four-parameter integrable deformation of the $\mathrm{SU}(2)$ principal chiral model corresponds to the Lukyanov model.

KeYwords: Integrable Field Theories, Sigma Models

ArXiv EPrint: 1707.08371 


\section{Contents}

1 Introduction 1

2 Bi-Yang-Baxter $\sigma$-model plus WZ term 2

2.1 Action 3

2.2 Lax pair 4

$\begin{array}{lll}3 & \text { TsT transformation } & 7\end{array}$

$\begin{array}{lll}3.1 & \text { On the action } & 7\end{array}$

$\begin{array}{ll}3.2 & \text { On the Lax pair } \\ 3.3 & 10\end{array}$

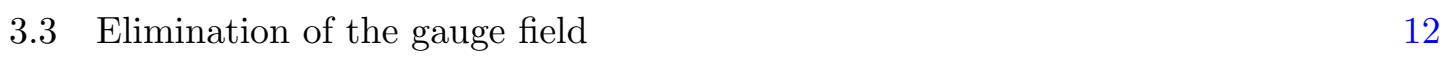

4 Equivalence with the Lukyanov model for $G=\mathrm{SU}(2) \quad 13$

5 Conclusion $\quad 15$

\section{Introduction}

In [1] Lukyanov constructed a novel four-parameter integrable deformation of the SU(2) principal chiral model $(\mathrm{PCM})$, which preserves a $\mathrm{U}(1) \times \mathrm{U}(1)$ subgroup of the original $\mathrm{SU}(2) \times \mathrm{SU}(2)$ global symmetry. This four-parameter model generalises $[1,2]$ a number of previously well-known theories:

- Fateev's two-parameter deformation of the SU(2) PCM [3]. This identification of the Fateev model as a special case of the Lukyanov model resolved the long-standing question of the integrability of the Fateev model.

- The SU(2) PCM plus the Wess-Zumino (WZ) term with arbitrary coefficient [4]. For a special value of this arbitrary coefficient one finds the conformal SU(2) WessZumino-Witten (WZW) model.

- The TsT transformation of the SU(2) WZW model, which can also be realised as a gauged WZW model for $(\mathrm{SU}(2) \times \mathrm{U}(1)) / \mathrm{U}(1)[5,6]$.

Lukyanov's model is defined by a metric and $B$-field. In the undeformed limit, the $B$-field vanishes and the metric is the one of the three-sphere. One may then ask if the full fourparameter deformation can be written as an action for a group-valued field $g \in \mathrm{SU}(2)$, and in turn generalised to arbitrary Lie group $G$.

Our aim in this paper is to answer these questions. To do this we will draw on a number of recent developments, many of which can trace their origins to Klimčík's YangBaxter $\sigma$-model $[7,8]$, a one-parameter integrable deformation of the PCM for a general group $G$, whose appellation reflects its dependence on a solution of the modified classical Yang-Baxter equation for $\mathfrak{g}=\operatorname{Lie}(G)$. 
The Yang-Baxter $\sigma$-model can be generalised to a two-parameter integrable deformation of the PCM, the bi-Yang-Baxter $\sigma$-model $[8,9]$, which also incorporates the oneparameter Yang-Baxter deformation of the symmetric space $\sigma$-model [10] for cosets of the type $(G \times G) / G_{\text {diag. }}$. Algebraically the two parameters manifest as $q$-deformations of the $G \times G$ symmetry, with an independent deformation parameter for each factor of the group $G[11]$ (see also $[12,10,13]$ ).

In [2] it was shown that the bi-Yang-Baxter $\sigma$-model for $G=\mathrm{SU}(2)$ is equivalent to Fateev's two-parameter deformation. This model does not have a non-trivial coupling to the $B$-field. In contrast the Lukyanov model does have such a coupling. As discussed above, for a certain choice of parameters this $B$-field corresponds to a WZ term. In [14] it was understood how to introduce such an anti-symmetric term for the Yang-Baxter $\sigma$-model while preserving classical integrability. This construction of the Yang-Baxter deformation of the PCM plus WZ term has been achieved for any Lie group $G$ and generalises the $\mathrm{SU}(2)$ case $[15,16]$.

The Yang-Baxter deformations of $[7,8,10]$ depend on a solution of the modified classical Yang-Baxter equation. However, they can also be defined in terms of a solution of the classical Yang-Baxter equation [17]. One of the simplest such solutions is when the $R$-matrix is abelian (i.e. when the generators from which it is built commute). In this case the homogeneous Yang-Baxter $\sigma$-model is equivalent to a TsT transformation [18-24].

In this paper we present a multi-parameter deformation of the PCM for a general group $G$ that incorporates each of the models introduced above. We furthermore construct a Lax pair that encodes its equations of motion, thereby demonstrating the classical integrability of the model. The number of deformation parameters depends on the group $G$. For $G=\mathrm{SU}(2)$ there are four parameters and in this case we explicitly demonstrate equivalence with Lukyanov's model [1]. Therefore, in this sense, the model is the generalisation of Lukyanov's model to arbitrary group $G$.

The construction of the model is split into two stages. In section 2 we consider a three-parameter integrable model: the bi-Yang-Baxter deformation of the PCM plus WZ term, generalising the construction of [14]. Generically this breaks the symmetry of the model from $G \times G$ to $\mathrm{U}(1)^{\operatorname{rank} G} \times \mathrm{U}(1)^{\operatorname{rank} G}$, i.e. the Cartan subgroup. We arrive at the Lagrangian and Lax pair for the multi-parameter deformation of the PCM in section 3 by implementing a general TsT transformation that mixes the Cartan generators of the two copies of $G$, which provides $(\operatorname{rank} G)^{2}$ additional parameters. For $G=\mathrm{SU}(2)$ the Cartan subgroup is one-dimensional and therefore there is one additional parameter. In section 4 we demonstrate the equivalence to Lukyanov's model. Finally we conclude in section 5 with comments and open questions.

\section{Bi-Yang-Baxter $\sigma$-model plus WZ term}

In this section we construct a three-parameter integrable deformation of the PCM. Two of these parameters correspond to those of the bi-Yang-Baxter $\sigma$-model while the third is the coupling to the WZ term. To obtain this integrable deformation of the PCM we employ on the following strategy. First of all, we shall view the PCM for a Lie group $G$ as the 
$(G \times G) / G_{\text {diag }}$ symmetric space $\sigma$-model, where $G_{\text {diag }}$ is the diagonal subgroup of $G \times G$. In the framework of integrable deformations, this perspective has been previously used in $[25,11]$. Secondly, the $G_{\text {diag }}$ gauge invariance will be realised by introducing a gauge field. In subsection 2.1 we start from an ansatz for the action with five free parameters and derive the corresponding equations of motion. We then determine the conditions for this action to define an integrable field theory in subsection 2.2. We show that a Lax pair exists provided the five parameters are fixed in terms of desired three deformation parameters.

\subsection{Action}

Let $G$ be a semi-simple real Lie group. We shall start from the action

$$
\begin{aligned}
S\left[g_{L, R}, A\right]= & -\int d^{2} \sigma \sum_{a, b=L, R} \operatorname{tr}\left[\left(j_{+}^{a}-A_{+}\right) O_{a b}\left(j_{-}^{b}-A_{-}\right)\right] \\
& +S_{\mathrm{WZ}, k}\left[g_{L}\right]-S_{\mathrm{WZ}, k}\left[g_{R}\right]-k \int d^{2} \sigma \operatorname{tr}\left[A_{-}\left(j_{+}^{L}-j_{+}^{R}\right)-A_{+}\left(j_{-}^{L}-j_{-}^{R}\right)\right],
\end{aligned}
$$

where $\sigma^{ \pm}$are light-cone coordinates. The fields $g_{L}$ and $g_{R}$ take values in the Lie group $G$ while the gauge field $A_{ \pm}$takes values in Lie algebra $\mathfrak{g}$. The left-invariant one-forms $j^{L}$ and $j^{R}$ are defined as $j^{a}=g_{a}^{-1} d g_{a}(a=L, R)$. The operators $O_{a b}$ are given by

$$
\begin{aligned}
O_{L L} & =\operatorname{Ad}_{g_{L}}^{-1}\left[\left(1+\eta_{L}^{2}\right) \frac{1+\mathcal{A}_{L} R}{1-\eta_{L}^{2} R^{2}}\right] \operatorname{Ad}_{g_{L}}, \\
O_{R R} & =\operatorname{Ad}_{g_{R}}^{-1}\left[\left(1+\eta_{R}^{2}\right) \frac{1+\mathcal{A}_{R} R}{1-\eta_{R}^{2} R^{2}}\right] \operatorname{Ad}_{g_{R}}, \\
O_{L R} & =O_{R L}=0,
\end{aligned}
$$

with $\operatorname{Ad}_{g}(x)=g x g^{-1}$ for $x \in \mathfrak{g}$. The operator $R$ is a non-split $R$-matrix on $\mathfrak{g}$. It is skewsymmetric and solves the modified classical Yang-Baxter equation on $\mathfrak{g}$, which means that for $x$ and $y$ in $\mathfrak{g}$ we have

$$
\begin{aligned}
& \operatorname{tr}(x R y)=-\operatorname{tr}(R x y), \\
& {[R x, R y]=R([R x, y]+[x, R y])+[x, y] .}
\end{aligned}
$$

Furthermore, we take $R$ to be a standard $R$-matrix, which implies that

$$
R^{3}=-R
$$

and that its non-trivial kernel is the Cartan subalgebra $\mathfrak{h}$ of $\mathfrak{g}$, i.e.

$$
R x=0, \quad \forall x \in \mathfrak{h} .
$$

The term $S_{\mathrm{WZ}, k}$ in (2.1) denotes the standard Wess-Zumino term,

$$
S_{\mathrm{WZ}, k}[g]=-k \int d^{2} \sigma d \xi \operatorname{tr}\left[g^{-1} \partial_{\xi} g\left[g^{-1} \partial_{+} g, g^{-1} \partial_{-} g\right]\right] .
$$


The presence of the WZ term indicates that the associated coupling should be quantised in the quantum theory. However, let us note that, in a mild abuse of notation, what we call $k$ is not the standard integer-valued level.

The action (2.1) is invariant under $G_{\text {diag }}$ gauge transformations,

$$
g_{L, R} \rightarrow g_{L, R} g_{0}, \quad A_{ \pm} \rightarrow g_{0}^{-1} \partial_{ \pm} g_{0}+g_{0}^{-1} A_{ \pm} g_{0},
$$

with $g_{0}\left(\sigma^{ \pm}\right)$taking values in $G$. This is so because $O_{a b}$ transforms as $O_{a b} \rightarrow \operatorname{Ad}_{g_{0}}^{-1} O_{a b} \operatorname{Ad}_{g_{0}}$ while $j_{ \pm}^{L}-j_{ \pm}^{R}$ and $j_{ \pm}^{L, R}-A_{ \pm}$have the homogeneous transformations $x \rightarrow \operatorname{Ad}_{g_{0}}^{-1} x$.

For the moment the coefficients $\mathcal{A}_{L, R}$ are free. The way they depend on $\eta_{L, R}$ and $k$ shall be fixed by imposing the existence of a Lax pair. The resulting dependence coincides with the analogous expressions in $[15,16,14]$.

Before we proceed to construct the Lax pair let us briefly illustrate the motivation for using a gauge field. To determine a Lax pair, we will have to explicitly invert operators such as $O_{a b}$. Without introducing a gauge field, the $G_{\text {diag }}$ gauge invariance would be ensured by making use of the projector onto the orthogonal complement of the diagonal subalgebra of $\mathfrak{g} \oplus \mathfrak{g}$ (see e.g. [25] for the bi-Yang-Baxter case). Such insertions of the projector operator make inverting the relevant operators in a tractable way substantially more difficult. As we shall see in the next subsection, the presence of the gauge field thus allows the inversion to be done in a simple way.

To construct a Lax pair we follow the method of [14] and start by determining the equations of motion. The equations of motion for the gauge field read

$$
J_{ \pm}^{L}+J_{ \pm}^{R}=0
$$

where

$$
\begin{array}{ll}
J_{-}^{L}=\left(O_{L L}+k\right)\left(j_{-}^{L}-A_{-}\right), & J_{+}^{L}=\left(O_{L L}^{t}-k\right)\left(j_{+}^{L}-A_{+}\right), \\
J_{-}^{R}=\left(O_{R R}-k\right)\left(j_{-}^{R}-A_{-}\right), & J_{+}^{R}=\left(O_{R R}^{t}+k\right)\left(j_{+}^{R}-A_{+}\right) .
\end{array}
$$

In these expressions, the operators $O_{L L}^{t}$ and $O_{R R}^{t}$ are obtained by taking the transpose of $O_{L L}$ and $O_{R R}$ respectively. This corresponds to flipping the sign of $R$.

The equations of motion for $g_{L}$ and $g_{R}$ are respectively given by

$$
\begin{aligned}
& D_{+} J_{-}^{L}+D_{-} J_{+}^{L}-2 k F_{-+}=0, \\
& D_{+} J_{-}^{R}+D_{-} J_{+}^{R}+2 k F_{-+}=0 .
\end{aligned}
$$

Here we have introduced covariant derivatives $D_{ \pm} x=\partial_{ \pm} x+\left[A_{ \pm}, x\right]$ and $F_{-+}$is the field strength of the gauge field,

$$
F_{-+}=\partial_{-} A_{+}-\partial_{+} A_{-}+\left[A_{-}, A_{+}\right]
$$

\section{$2.2 \quad$ Lax pair}

To proceed we treat the equations of motion for the gauge field (2.8) separately to those for $g_{a}$. In particular, as is typical for constraint equations, they will not be determined by the zero curvature condition for the Lax pair. 
The currents $J_{ \pm}^{R}$ can be obtained from the equations of motion for the gauge field (2.8), and are, on-shell, just the negative of $J_{ \pm}^{L}$. We shall therefore focus on the currents $J_{ \pm}^{L}$, their equation of motion (2.10a) and the Maurer-Cartan equation,

$$
\partial_{-} j_{+}^{L}-\partial_{+} j_{-}^{L}+\left[j_{-}^{L}, j_{+}^{L}\right]=0 .
$$

From now on, we explicitly use the relation $R^{3}=-R$ in order to write all operators, such as $O_{L L}$, as a linear combination of $\Pi=1+R^{2}, R$ and $R^{2}$. The operator $\Pi$ is the projector on the Cartan subalgebra $\mathfrak{h}$. To do this one can use the relations

$$
\begin{gathered}
\Pi=1+R^{2}, \quad \Pi^{2}=\Pi, \quad \Pi R=R \Pi=0, \\
\left(a \Pi+b R+c R^{2}\right)^{-1}=a^{-1} \Pi+\frac{1}{b^{2}+c^{2}}\left(-b R+c R^{2}\right) .
\end{gathered}
$$

Now expressing the currents $j_{ \pm}^{L}$ in terms of $J_{ \pm}^{L}$ and $A$ using (2.9a) and (2.12b) leads to

$$
j_{ \pm}^{L}=\left(a_{ \pm} \Pi_{L L}+b_{ \pm} R_{L L}+d_{ \pm} R_{L L}^{2}\right) J_{ \pm}^{L}+A_{ \pm}
$$

where we make use of a general notation for operators dressed by the adjoint action, e.g. $\Pi_{L L}=\operatorname{Ad}_{g_{L}}^{-1} \Pi \operatorname{Ad}_{g_{L}}$. The coefficients $a_{ \pm}, b_{ \pm}$and $d_{ \pm}$are given by

$$
a_{ \pm}=\frac{1}{1+\eta_{L}^{2} \mp k}, \quad b_{ \pm}=\frac{ \pm \mathcal{A}_{L}}{\mathcal{A}_{L}^{2}+(1 \mp k)^{2}}, \quad d_{ \pm}=-\frac{1 \mp k}{\mathcal{A}_{L}^{2}+(1 \mp k)^{2}}
$$

These coefficients satisfy the relations

$$
-b_{+} d_{-}-b_{-} d_{+}=\frac{1}{2}\left(b_{+}+b_{-}\right), \quad b_{+} b_{-}-d_{+} d_{-}=\frac{1}{2}\left(d_{+}+d_{-}\right) .
$$

Note that the analogous expressions for the right currents are obtained from the left ones by the replacement rule $\left(L, \eta_{L}, \mathcal{A}_{L}, k\right) \rightarrow\left(R, \eta_{R}, \mathcal{A}_{R},-k\right)$.

Let us denote the left-hand side of the Maurer-Cartan equation (2.11) as $\mathrm{MC}_{L}$. Starting from (2.13) we may rewrite $\mathrm{MC}_{L}$ as

$$
\begin{aligned}
\mathrm{MC}_{L}= & \left(\frac{a_{+}-a_{-}}{2} \Pi_{L L}+\frac{b_{+}-b_{-}}{2} R_{L L}+\frac{d_{+}-d_{-}}{2} R_{L L}^{2}\right)\left(D_{-} J_{+}^{L}+D_{+} J_{-}^{L}\right)+F_{-+} \\
+ & \left(\frac{a_{+}+a_{-}}{2} \Pi_{L L}+\frac{b_{+}+b_{-}}{2} R_{L L}+\frac{d_{+}+d_{-}}{2} R_{L L}^{2}\right)\left(D_{-} J_{+}^{L}-D_{+} J_{-}^{L}\right) \\
+ & \left(-\left(b_{+} b_{-}+d_{+} d_{-}+d_{+} a_{-}+a_{+} d_{-}\right) \Pi_{L L}\right. \\
& \left.-\left(b_{+} d_{-}+d_{+} b_{-}\right) R_{L L}+\left(b_{+} b_{-}-d_{+} d_{-}\right) R_{L L}^{2}\right)\left[J_{-}^{L}, J_{+}^{L}\right] \\
+ & b_{+}\left(a_{-}+d_{-}\right) \Pi_{L L}\left[J_{-}^{L}, R_{L L} J_{+}^{L}\right]+b_{-}\left(a_{+}+d_{+}\right) \Pi_{L L}\left[R_{L L} J_{-}^{L}, J_{+}^{L}\right] .
\end{aligned}
$$

If we choose $\mathcal{A}_{L}$ as in $[15,16,14]$,

$$
\mathcal{A}_{L}^{2}=\eta_{L}^{2}\left(1-\frac{k^{2}}{1+\eta_{L}^{2}}\right)
$$


then the coefficients $a_{ \pm}, b_{ \pm}$and $d_{ \pm}$satisfy the following relations

$$
\begin{aligned}
b_{+}\left(a_{-}+d_{-}\right) & =b_{-}\left(a_{+}+d_{+}\right), \\
-\left(b_{+} b_{-}+d_{+} d_{-}+d_{+} a_{-}+a_{+} d_{-}\right) & =\frac{1}{2}\left(a_{+}+a_{-}\right) .
\end{aligned}
$$

This choice has the following consequences. Let us start with (2.18a). Since the standard $R$-matrix satisfies (see for instance [14])

$$
\Pi([R x, y]+[x, R y])=0 \quad \forall x, y \in \mathfrak{g},
$$

the last line in (2.16) vanishes. The next step is to use (2.18b) and (2.15) to combine the third line of (2.16) with the second one. Finally, we use the equation of motion (2.10a) in the first line of (2.16). Following these steps we obtain

$$
\begin{aligned}
\mathrm{MC}_{L}= & \left(\left[1+k\left(a_{+}-a_{-}\right)\right] \Pi_{L L}+k\left(b_{+}-b_{-}\right) R_{L L}+\left[-1+k\left(d_{+}-d_{-}\right)\right] R_{L L}^{2}\right) F_{-+} \\
& +\left(\frac{a_{+}+a_{-}}{2} \Pi_{L L}+\frac{b_{+}+b_{-}}{2} R_{L L}+\frac{d_{+}+d_{-}}{2} R_{L L}^{2}\right)\left(D_{-} J_{+}^{L}-D_{+} J_{-}^{L}+\left[J_{-}^{L}, J_{+}^{L}\right]\right) .
\end{aligned}
$$

The condition (2.17) implies that the operators appearing in the first and second lines of (2.20) are proportional, with the relative coefficient being equal to $\left(1+k^{2}+\mathcal{A}_{L}^{2}\right)$. Furthermore, these operators are invertible. Therefore, on-shell, the equation $\mathrm{MC}_{L}=0$ is equivalent to

$$
D_{-} J_{+}^{L}-D_{+} J_{-}^{L}+\left[J_{-}^{L}, J_{+}^{L}\right]+\left(1+k^{2}+\mathcal{A}_{L}^{2}\right) F_{-+}=0 .
$$

Proceeding in the same way for the right currents, choosing in particular

$$
\mathcal{A}_{R}^{2}=\eta_{R}^{2}\left(1-\frac{k^{2}}{1+\eta_{R}^{2}}\right)
$$

one similarly arrives at

$$
D_{-} J_{+}^{R}-D_{+} J_{-}^{R}+\left[J_{-}^{R}, J_{+}^{R}\right]+\left(1+k^{2}+\mathcal{A}_{R}^{2}\right) F_{-+}=0 .
$$

We now take the sum of (2.21) and (2.23) and use the equations of motion for the gauge field (2.8) to express the field strength $F_{-+}$in terms of $\left[J_{-}^{L}, J_{+}^{L}\right]$. We then use this expression for $F_{-+}$in (2.10a) and (2.21) to obtain

$$
\begin{aligned}
F_{-+}-\frac{1}{2 k}\left(\alpha_{+}-\alpha_{-}\right)\left[J_{-}^{L}, J_{+}^{L}\right] & =0, \\
D_{+} J_{-}^{L}+D_{-} J_{+}^{L}-\left(\alpha_{+}-\alpha_{-}\right)\left[J_{-}^{L}, J_{+}^{L}\right] & =0, \\
D_{+} J_{-}^{L}-D_{-} J_{+}^{L}-\left(\alpha_{+}+\alpha_{-}\right)\left[J_{-}^{L}, J_{+}^{L}\right] & =0,
\end{aligned}
$$

with

$$
\alpha_{+}=\frac{-\mathcal{A}_{L}^{2}+\mathcal{A}_{R}^{2}-4 k}{2\left(2\left(1+k^{2}\right)+\mathcal{A}_{L}^{2}+\mathcal{A}_{R}^{2}\right)}, \quad \alpha_{-}=\frac{-\mathcal{A}_{L}^{2}+\mathcal{A}_{R}^{2}+4 k}{2\left(2\left(1+k^{2}\right)+\mathcal{A}_{L}^{2}+\mathcal{A}_{R}^{2}\right)}
$$


To construct a Lax pair let us redefine the gauge field as

$$
\widehat{A}_{ \pm}=A_{ \pm}+\alpha_{ \pm} J_{ \pm}^{L}
$$

The equations (2.24) are then equivalent to

$$
\begin{aligned}
\widehat{F}_{-+}=F_{-+}-\alpha_{+} \alpha_{-}\left[J_{-}^{L}, J_{+}^{L}\right] & =-G^{2}\left[J_{-}^{L}, J_{+}^{L}\right], \\
\widehat{D}_{+} J_{-}^{L}+\widehat{D}_{-} J_{+}^{L} & =0, \\
\widehat{D}_{+} J_{-}^{L}-\widehat{D}_{-} J_{+}^{L} & =0,
\end{aligned}
$$

where $\widehat{D}$ are covariant derivatives with respect to $\widehat{A}$ and

$$
G^{2}=\frac{\left(4+\left(\mathcal{A}_{L}+\mathcal{A}_{R}\right)^{2}\right)\left(4+\left(\mathcal{A}_{L}-\mathcal{A}_{R}\right)^{2}\right)}{4\left(2\left(1+k^{2}\right)+\mathcal{A}_{L}^{2}+\mathcal{A}_{R}^{2}\right)^{2}} .
$$

The equations (2.27) are equivalent to the flatness of the Lax pair

$$
\mathcal{L}_{ \pm}(\lambda)=\widehat{A}_{ \pm}+G \lambda^{ \pm 1} J_{ \pm}^{L}
$$

where $\lambda$ is a spectral parameter. We have therefore shown that the action (2.1) defines an integrable model with $\mathcal{A}_{L, R}$ given by (2.17) and (2.22).

\section{TsT transformation}

The three-parameter deformation of the PCM constructed in section 2 breaks the global $G \times G$ symmetry of the action. As a consequence of the property (2.5) the symmetry that remains is the Cartan subgroup specified by the kernel of the operator $R$. By implementing TsT transformations $[26,5,6]$ on the corresponding shift isometries we are able to introduce additional deformation parameters while preserving integrability [27-29]. In this section we perform a general TsT transformation with each of the two shift isometries coming from a different copy of $G$.

\subsection{On the action}

Our starting point is the action (2.1). As shown in subsection 2.2 the equations of motion for $g_{L}$ and $g_{R}$ and the Maurer-Cartan equations follow from a Lax pair if $\mathcal{A}_{L}$ and $\mathcal{A}_{R}$ are fixed in terms of $\eta_{L}, \eta_{R}$ and $k$ as

$$
\mathcal{A}_{L}^{2}=\eta_{L}^{2}\left(1-\frac{k^{2}}{1+\eta_{L}^{2}}\right), \quad \mathcal{A}_{R}^{2}=\eta_{R}^{2}\left(1-\frac{k^{2}}{1+\eta_{R}^{2}}\right)
$$

For $\eta_{L}=\eta_{R}=0$ the symmetry of the action is $G \times G$, which is broken to the Cartan subgroup $\mathrm{U}(1)^{\operatorname{rank} G} \times \mathrm{U}(1)^{\operatorname{rank} G}$ for generic values of the deformation parameters. To implement the TsT transformations in the Cartan directions we start by making the corresponding shift isometries manifest. To this end we parameterise $(a=L, R)$

$$
g_{a}=\exp \left(x_{a}\right) \tilde{g}_{a}, \quad \tilde{g}_{a} \in G, \quad x_{a} \in \mathfrak{h},
$$


such that

$$
j_{ \pm}^{a}=\tilde{\jmath}_{ \pm}^{a}+\operatorname{Ad}_{\tilde{g}_{a}}^{-1} \partial_{ \pm} x_{a}
$$

where $\tilde{j}^{a}$ is the left-invariant one-form associated with $\tilde{g}_{a}$, i.e. $\tilde{j}^{a}=\tilde{g}_{a}^{-1} d \tilde{g}_{a}$. It is important to note that the parameterisation (3.2) introduces a new left-acting Cartan gauge symmetry

$$
x_{a} \rightarrow x_{a}+\xi_{a}, \quad \tilde{g}_{a} \rightarrow \exp \left(-\xi_{a}\right) \tilde{g}_{a} .
$$

As we will see this symmetry survives the TsT transformation (up to potential total derivatives). Therefore for now we leave it unfixed, using the $x_{a}$ coordinates to implement the deformation, and fix it only at the end.

Defining the combinations

$$
l_{ \pm}^{a}=\operatorname{Ad}_{\tilde{g}_{a}}\left(\tilde{\jmath}_{ \pm}^{a}-A_{ \pm}\right),
$$

which are invariant under the original $G_{\text {diag }}$ gauge transformations (2.7), and the rescaled projections of $l_{ \pm}^{a}$ onto the Cartan subalgebra (recall that $\Pi=1+R^{2}$ is the projector onto $\mathfrak{h}$ )

$$
L_{ \pm}^{L}=\left(1+\eta_{L}^{2} \pm k\right) \Pi l_{ \pm}^{L}, \quad L_{ \pm}^{R}=\left(1+\eta_{R}^{2} \mp k\right) \Pi l_{ \pm}^{R},
$$

we use (2.4) and (2.5) to rewrite the action (2.1) in the form

$$
\begin{aligned}
S\left[\tilde{g}_{L, R}, x_{L, R}, A\right]= & -\int d^{2} \sigma \operatorname{tr}\left[l_{+}^{L} \mathcal{O}_{L} l_{-}^{L}+L_{-}^{L} \partial_{+} x_{L}+L_{+}^{L} \partial_{-} x_{L}+\left(1+\eta_{L}^{2}\right) \partial_{+} x_{L} \partial_{-} x_{L}\right] \\
& -\int d^{2} \sigma \operatorname{tr}\left[l_{+}^{R} \mathcal{O}_{R} l_{-}^{R}+L_{-}^{R} \partial_{+} x_{R}+L_{+}^{R} \partial_{-} x_{R}+\left(1+\eta_{R}^{2}\right) \partial_{+} x_{R} \partial_{-} x_{R}\right] \\
& +S_{\mathrm{WZ}, k}\left[\tilde{g}_{L}\right]-S_{\mathrm{WZ}, k}\left[\tilde{g}_{R}\right]-k \int d^{2} \sigma \operatorname{tr}\left[A_{-}\left(\tilde{\jmath}_{+}^{L}-\tilde{\jmath}_{+}^{R}\right)-A_{+}\left(\tilde{\jmath}_{-}^{L}-\tilde{\jmath}_{-}^{R}\right)\right],
\end{aligned}
$$

where the operators $\mathcal{O}_{L, R}$ are given by

$$
\mathcal{O}_{L, R}=1+\mathcal{A}_{L, R} R+\eta_{L, R}^{2} \Pi \text {. }
$$

To implement the TsT transformation we first T-dualise $x_{L} \rightarrow \tilde{x}_{L}$, then perform the shift $x_{R}=\hat{x}_{R}+\omega \tilde{x}_{L}$, where $\omega$ is a constant linear operator on the Cartan subalgebra $\mathfrak{h}$ containing $(\operatorname{rank} G)^{2}$ additional parameters, and finally implement the reverse T-duality $\tilde{x}_{L} \rightarrow \hat{x}_{L}$. Eventually we arrive at the action

$$
\begin{aligned}
& S_{\omega}\left[\tilde{g}_{L, R}, \hat{x}_{L, R}, A\right]=-\int d^{2} \sigma \operatorname{tr} {\left[l_{+}^{L} \mathcal{O}_{L} l_{-}^{L}-\left(1+\eta_{R}^{2}\right) L_{+}^{L} \omega^{t} \widetilde{\mathcal{O}}^{-1} \omega L_{-}^{L}\right.} \\
&\left.+L_{+}^{L} \mathcal{O}^{-1} \partial_{-} \hat{x}_{L}+L_{-}^{L} \mathcal{O}^{-1} \partial_{+} \hat{x}_{L}+\left(1+\eta_{L}^{2}\right) \partial_{+} \hat{x}_{L} \mathcal{O}^{-1} \partial_{-} \hat{x}_{L}\right] \\
&-\int d^{2} \sigma \operatorname{tr}\left[l_{+}^{R} \mathcal{O}_{R} l_{-}^{R}-\left(1+\eta_{L}^{2}\right) L_{+}^{R} \omega \mathcal{O}^{-1} \omega^{t} L_{-}^{R}\right. \\
&+\left.L_{+}^{R} \widetilde{\mathcal{O}}^{-1} \partial_{-} \hat{x}_{R}+L_{-}^{R} \widetilde{\mathcal{O}}^{-1} \partial_{+} \hat{x}_{R}+\left(1+\eta_{R}^{2}\right) \partial_{+} \hat{x}_{R} \widetilde{\mathcal{O}}^{-1} \partial_{-} \hat{x}_{R}\right] \\
&+\int d^{2} \sigma \operatorname{tr}\left[\left(L_{+}^{L}+\left(1+\eta_{L}^{2}\right) \partial_{+} \hat{x}_{L}\right) \mathcal{O}^{-1} \omega^{t}\left(L_{-}^{R}+\left(1+\eta_{R}^{2}\right) \partial_{-} \hat{x}_{R}\right)\right. \\
&\left.\quad-\left(L_{+}^{R}+\left(1+\eta_{R}^{2}\right) \partial_{+} \hat{x}_{R}\right) \widetilde{\mathcal{O}}^{-1} \omega\left(L_{-}^{L}+\left(1+\eta_{L}^{2}\right) \partial_{-} \hat{x}_{L}\right)\right] \\
&+S_{\mathrm{WZ}, k}\left[\tilde{g}_{L}\right]-S_{\mathrm{WZ}, k}\left[\tilde{g}_{R}\right]-k \int d^{2} \sigma \operatorname{tr}\left[A_{-}\left(\tilde{\jmath}_{+}^{L}-\tilde{\jmath}_{+}^{R}\right)-A_{+}\left(\tilde{\jmath}_{-}^{L}-\tilde{\jmath}_{-}^{R}\right)\right],
\end{aligned}
$$


with

$$
\mathcal{O}=1+\left(1+\eta_{L}^{2}\right)\left(1+\eta_{R}^{2}\right) \omega^{t} \omega, \quad \widetilde{\mathcal{O}}=1+\left(1+\eta_{L}^{2}\right)\left(1+\eta_{R}^{2}\right) \omega \omega^{t}
$$

Note that $\mathcal{O}$ and $\widetilde{\mathcal{O}}$ are related as follows

$$
\omega^{t} \widetilde{\mathcal{O}}^{-1} \omega=\left(\omega^{t} \widetilde{\mathcal{O}}^{-1} \omega\right)^{t}=\mathcal{O}^{-1} \omega^{t} \omega, \quad \omega \mathcal{O}^{-1} \omega^{t}=\left(\omega \mathcal{O}^{-1} \omega^{t}\right)^{t}=\widetilde{\mathcal{O}}^{-1} \omega \omega^{t} .
$$

In order to recast the action (3.9) in a form generalising (2.1) we parameterise

$$
\tilde{g}_{a}=\exp \left(y_{a}\right) \hat{g}_{a}, \quad \hat{g}_{a} \in G, \quad y_{a} \in \mathfrak{h} .
$$

Setting

$$
y_{L}=-\left(1-k^{2} \omega^{t} \omega\right)^{-1}\left(\hat{x}_{L}+k \omega^{t} \hat{x}_{R}\right), \quad y_{R}=-\left(1-k^{2} \omega \omega^{t}\right)^{-1}\left(\hat{x}_{R}+k \omega \hat{x}_{L}\right),
$$

we find that the $\hat{x}_{a}$ dependence drops out of the action up to the total derivative

$$
-k^{2}\left[\partial_{+} \hat{x}_{L}\left(1-k^{2} \omega^{t} \omega\right)^{-1} \omega^{t} \partial_{-} \hat{x}_{R}-\partial_{-} \hat{x}_{L}\left(1-k^{2} \omega^{t} \omega\right)^{-1} \omega^{t} \partial_{+} \hat{x}_{R}\right],
$$

which we also drop. We expect to be able to remove the dependence on $\hat{x}_{a}$ in this way as a consequence of the left-acting Cartan gauge invariance (3.4). As foreseen this symmetry survives the TsT transformation up to potential total derivatives that we ignored in the T-dualisations.

Renaming $\hat{g}_{a}$ as $g_{a}$, we are finally left with the action

$$
\begin{aligned}
S_{\omega}\left[g_{L, R}, A\right]= & -\int d^{2} \sigma \sum_{a, b=L, R} \operatorname{tr}\left[\left(j_{+}^{a}-A_{+}\right) O_{a b, \omega}\left(j_{-}^{b}-A_{-}\right)\right] \\
& +S_{\mathrm{WZ}, k}\left[g_{L}\right]-S_{\mathrm{WZ}, k}\left[g_{R}\right]-k \int d^{2} \sigma \operatorname{tr}\left[A_{-}\left(j_{+}^{L}-j_{+}^{R}\right)-A_{+}\left(j_{-}^{L}-j_{-}^{R}\right)\right],
\end{aligned}
$$

where the dressed operators are now given by

$$
\begin{aligned}
O_{L L, \omega} & =\operatorname{Ad}_{g_{L}}^{-1}\left[1+\mathcal{A}_{L} R+\left(\eta_{L}^{2}-\left(1+\eta_{R}^{2}\right)\left(1+\eta_{L}^{2}+k\right)\left(1+\eta_{L}^{2}-k\right)\left(\omega^{t} \widetilde{\mathcal{O}}^{-1} \omega\right)\right) \Pi\right] \operatorname{Ad}_{g_{L}} \\
O_{R R, \omega} & =\operatorname{Ad}_{g_{R}}^{-1}\left[1+\mathcal{A}_{R} R+\left(\eta_{R}^{2}-\left(1+\eta_{L}^{2}\right)\left(1+\eta_{R}^{2}+k\right)\left(1+\eta_{R}^{2}-k\right)\left(\omega \mathcal{O}^{-1} \omega^{t}\right)\right) \Pi\right] \operatorname{Ad}_{g_{R}}, \\
O_{L R, \omega} & =\operatorname{Ad}_{g_{L}}^{-1}\left[\left(1+\eta_{L}^{2}+k\right)\left(1+\eta_{R}^{2}+k\right)\left(\mathcal{O}^{-1} \omega^{t}\right) \Pi\right] \operatorname{Ad}_{g_{R}}, \\
O_{R L, \omega} & =-\operatorname{Ad}_{g_{R}}^{-1}\left[\left(1+\eta_{L}^{2}-k\right)\left(1+\eta_{R}^{2}-k\right)\left(\widetilde{\mathcal{O}}^{-1} \omega\right) \Pi\right] \operatorname{Ad}_{g_{L}}
\end{aligned}
$$

with $\mathcal{A}_{L, R}$ defined in terms of $\eta_{L, R}$ and $k$ in (3.1), $\mathcal{O}$ and $\widetilde{\mathcal{O}}$ given in (3.10) and we recall that $\omega$ is an arbitrary constant linear operator on $\mathfrak{h}$. As we will shortly demonstrate via the existence of a Lax pair this multi-parameter deformation of the PCM is integrable.

Before we do so, let us briefly consider various limits of (3.14) in order to gain a better understanding of the model. First we note that, as expected, upon setting $\omega=0$ we recover the three-parameter deformation of section 2, i.e. the bi-Yang-Baxter deformation of the PCM plus WZ term. Additionally setting either $\eta_{L}$ or $\eta_{R}$ to zero we expect to find the one-parameter Yang-Baxter deformation of the PCM plus WZ term constructed in [14]. The model of [14] depends on a single field $g \in G$ and hence to explicitly check this 
relation we integrate out the gauge field. This is done in section 3.3 for the multi-parameter deformation (3.14), with the resulting action given in (3.36). The latter only depends on $g_{L}$ and $g_{R}$ through the combination $g=g_{L} g_{R}^{-1}$ as a consequence of the gauge symmetry (2.7), and indeed setting $\omega=\eta_{R}=0$ we recover the model of [14].

It is also interesting to consider the limit $k=0$, that is when the WZ term is no longer present. In this case we can rewrite the deformed action in a form familiar in the context of Yang-Baxter deformations

$$
\left.S_{\omega}\left[g_{L, R}, A\right]\right|_{k=0}=-\int d^{2} \sigma \operatorname{tr}\left[\left(j_{+}^{L}-A_{+}, j_{+}^{R}-A_{+}\right) \cdot \mathscr{O} \cdot\left(j_{-}^{L}-A_{-}, j_{-}^{R}-A_{-}\right)^{t}\right],
$$

where the operator $\mathscr{O}$ is given by

$$
\mathscr{O}=\left(\begin{array}{cc}
\sqrt{1+\eta_{L}^{2}} & 0 \\
0 & \sqrt{1+\eta_{R}^{2}}
\end{array}\right) \cdot \frac{1}{1-\mathscr{R}_{g_{L, R}}} \cdot\left(\begin{array}{cc}
\sqrt{1+\eta_{L}^{2}} & 0 \\
0 & \sqrt{1+\eta_{R}^{2}}
\end{array}\right),
$$

which in turn is defined in terms of a linear operator $\mathscr{R}$ acting on $\mathfrak{g} \oplus \mathfrak{g}$

$$
\begin{aligned}
\mathscr{R}_{g_{L, R}} & =\left(\begin{array}{cc}
\operatorname{Ad}_{g_{L}}^{-1} & 0 \\
0 & \operatorname{Ad}_{g_{R}}^{-1}
\end{array}\right) \cdot \mathscr{R} \cdot\left(\begin{array}{cc}
\operatorname{Ad}_{g_{L}} & 0 \\
0 & \operatorname{Ad}_{g_{R}}
\end{array}\right) \\
\mathscr{R} & =\left(\begin{array}{cc}
\eta_{L} R & \sqrt{\left(1+\eta_{L}^{2}\right)\left(1+\eta_{R}^{2}\right)} \omega^{t} \Pi \\
-\sqrt{\left(1+\eta_{L}^{2}\right)\left(1+\eta_{R}^{2}\right)} \omega \Pi & \eta_{R} R
\end{array}\right) .
\end{aligned}
$$

For all $X=\left(x_{L}, x_{R}\right)^{t}$ and $Y=\left(y_{L}, y_{R}\right)^{t}$ in $\mathfrak{g} \oplus \mathfrak{g}$ the operator $\mathscr{R}$ satisfies the modified classical Yang-Baxter equation

$$
[\mathscr{R} X, \mathscr{R} Y]-\mathscr{R}[\mathscr{R} X, Y]-\mathscr{R}[X, \mathscr{R} Y]=\left(\begin{array}{c}
\eta_{L}^{2}\left[x_{L}, y_{L}\right] \\
\eta_{R}^{2}\left[x_{R}, y_{R}\right]
\end{array}\right) .
$$

Note that the right-hand side of (3.19) is independent of $\omega$ and hence if we additionally set $\eta_{L}=\eta_{R}=0$ the operator $\mathscr{R}$ satisfies the classical Yang-Baxter equation. In this case we are left with the homogeneous Yang-Baxter deformation of the PCM with an abelian $R$-matrix, which is equivalent to a series of TsT transformations [18-24]. Alternatively we may set $\omega=0$, in which case we recover the bi-Yang-Baxter sigma model of $[8,9,25,11]$. Finally, if $\eta_{L}^{2}=\eta_{R}^{2}=\eta^{2}$ and $\left(\eta+\eta^{-1}\right)^{2} \omega \omega^{t}=\left(\eta+\eta^{-1}\right)^{2} \omega^{t} \omega=1$ then the operator $\mathscr{R}$ satisfies

$$
\eta^{-2} \mathscr{R}^{2}=-1
$$

Therefore, $\eta^{-1} \mathscr{R}$ defines a complex structure on $G \times G$. Yang-Baxter deformations based on complex structures have been explored in [30] and typically give rise to particularly simple models.

\subsection{On the Lax pair}

The Lax pair (2.29) for the three-parameter model described by the action (2.1) is given by

$$
\mathcal{L}_{ \pm}(\lambda)=A_{ \pm}+\alpha_{ \pm} J_{ \pm}^{L}+G \lambda^{ \pm 1} J_{ \pm}^{L}
$$


with the parameters $\alpha_{ \pm}, G$ given in (2.25) and (2.28) respectively. The zero-curvature equation for $\mathcal{L}_{ \pm}(\lambda)$ implies the equations of motion (2.10) and Maurer-Cartan equations, (2.21) and (2.23). Furthermore, it should be supplemented with the equations of motion for the gauge field (2.8), which are constraint equations fixing the gauge field in terms of the group fields.

The Lax pair (3.21) and the constraint equations (2.8) are written in terms of the currents $J_{ \pm}^{a}$ and the gauge field $A_{ \pm}$, where the dependence on $g_{a}$ is contained within the former. Therefore, to determine the Lax pair and constraint equations for the TsT transformed model we just implement the transformation on $J_{ \pm}^{a}$, which gives the TsT transformed currents. It then follows that the Lax pair for the model described by the TsT transformed action (3.14) has the same form (3.21) as it had before transformation, only now with $J_{ \pm}^{a}$ given by TsT transformed expressions for the currents. The same holds for the constraint equations (2.8).

To construct the currents of the TsT transformed model we start from those of the three-parameter model defined by (2.9). Using the parameterisation (3.2) these can be written as

$$
J_{ \pm}^{L}=\tilde{J}_{ \pm}^{L}+A d_{\tilde{g}_{L}}^{-1}\left(1 \mp k+\eta_{L}^{2}\right) \partial_{ \pm} x_{L}, \quad J_{ \pm}^{R}=\tilde{J}_{ \pm}^{R}+A d_{\tilde{g}_{R}}^{-1}\left(1 \pm k+\eta_{R}^{2}\right) \partial_{ \pm} x_{R},
$$

where the $\tilde{J}_{ \pm}^{a}$ are simply obtained from $J_{ \pm}^{a}$ by the replacement $g_{a} \rightarrow \tilde{g}_{a}$. The currents $J_{ \pm}^{a}$, and thus the Lax pair, equations of motion and Maurer-Cartan equations, only depend on derivatives of the Cartan subalgebra valued fields $x_{a}$. Then, following, for example, [28], we track the fate of the derivatives $\partial_{ \pm} x_{a}$ through the TsT transformation.

In the first step, that is under the T-duality $x_{L} \rightarrow \tilde{x}_{L}$, one has

$$
\partial_{ \pm} x_{L}=-\frac{1}{1+\eta_{L}^{2}}\left(L_{ \pm}^{L} \mp \partial_{ \pm} \tilde{x}_{L}\right)
$$

where $L_{ \pm}^{a}$ are defined in (3.6). The second step is a translation of $x_{R}$ and implies

$$
\hat{x}_{R}=x_{R}-\omega \tilde{x}_{L} \quad \Rightarrow \quad \partial_{ \pm} x_{R}=\partial_{ \pm} \hat{x}_{R}+\omega \partial_{ \pm} \tilde{x}_{L} .
$$

Finally, the second T-duality, $\tilde{x}_{L} \rightarrow \hat{x}_{L}$, gives

$$
\partial_{ \pm} \tilde{x}_{L}=\mathcal{O}^{-1}\left( \pm L_{ \pm}^{L}-\left(1+\eta_{L}^{2}\right)\left(\omega^{t}\left(L_{ \pm}^{R}+\left(1+\eta_{R}^{2}\right) \partial_{ \pm} \hat{x}_{R}\right) \pm \partial_{ \pm} \hat{x}_{L}\right)\right)
$$

where $\mathcal{O}$ is defined in (3.10). Once the TsT transformation is performed, we fix the gauge $\hat{x}_{L}=\hat{x}_{R}=0$ using the gauge symmetry (3.4). Recall that, as discussed in subsection 3.1, this symmetry survives the TsT transformation up to total derivatives, which do not contribute to the equations of motion. For this gauge choice the expressions for $\partial_{ \pm} x_{a}$ in (3.23) and (3.24) become

$$
\begin{aligned}
& \partial_{ \pm} x_{L}=-\left(1+\eta_{R}^{2}\right) \mathcal{O}^{-1} \omega^{t} \omega L_{ \pm}^{L} \mp \mathcal{O}^{-1} \omega^{t} L_{ \pm}^{R} \\
& \partial_{ \pm} x_{R}=-\left(1+\eta_{L}^{2}\right) \tilde{\mathcal{O}}^{-1} \omega \omega^{t} L_{ \pm}^{R} \pm \tilde{\mathcal{O}}^{-1} \omega L_{ \pm}^{L}
\end{aligned}
$$

Substituting into (3.22) we find expressions for TsT transformed currents $J_{ \pm}^{a}$ as a function of the field $\tilde{g}_{a} \in G$. Finally, to match with the action (3.14) we rename $\tilde{g}_{a}$ as $g_{a}$, after 
which these currents are expressed as follows

$$
\begin{aligned}
& J_{-}^{L}=\left(O_{L L, \omega}+k\right)\left(j_{-}^{L}-A_{-}\right)+O_{L R, \omega}\left(j_{-}^{R}-A_{-}\right), \\
& J_{+}^{L}=\left(O_{L L, \omega}^{t}-k\right)\left(j_{+}^{L}-A_{+}\right)+O_{R L, \omega}^{t}\left(j_{+}^{R}-A_{+}\right), \\
& J_{-}^{R}=\left(O_{R R, \omega}-k\right)\left(j_{-}^{R}-A_{-}\right)+O_{R L, \omega}\left(j_{-}^{L}-A_{-}\right), \\
& J_{+}^{R}=\left(O_{R R, \omega}^{t}+k\right)\left(j_{+}^{R}-A_{+}\right)+O_{L R, \omega}^{t}\left(j_{+}^{L}-A_{+}\right),
\end{aligned}
$$

where the various operators are defined in (3.15). Therefore, the Lax pair of the TsT transformed model (3.14) takes the form (3.21) with $J_{ \pm}^{a}$ now given by (3.27). As before, this Lax pair should be supplemented by constraint equations of the form (2.8), again with $J_{ \pm}^{a}$ given by (3.27). Note that these results also follow from direct computation, in the spirit of subsection 2.2, starting from the action (3.14).

\subsection{Elimination of the gauge field}

Let us now eliminate the gauge field from the action (3.14). The resulting action will be the starting point in the next section for the comparison with Lukyanov's model.

The equations of motion for the gauge field (2.8) and the definitions of $J_{ \pm}^{a}$ given in (3.27) can be used to write the left-invariant currents $j_{ \pm}^{a}$ as

$$
\begin{array}{ll}
j_{-}^{L}-A_{-}=\mathcal{Q}_{L} J_{-}^{L}, & j_{-}^{R}-A_{-}=-\mathcal{Q}_{R} J_{-}^{L}, \\
j_{+}^{L}-A_{+}=\mathcal{P}_{L}^{t} J_{+}^{L}, & j_{+}^{R}-A_{+}=-\mathcal{P}_{R}^{t} J_{+}^{L},
\end{array}
$$

where $\mathcal{Q}_{L}, \mathcal{Q}_{R}, \mathcal{P}_{L}$ and $\mathcal{P}_{R}$ are the following operators

$$
\begin{aligned}
& \mathcal{Q}_{L}=\left(O_{L L, \omega}+k-O_{L R, \omega}\left(O_{R R, \omega}-k\right)^{-1} O_{R L, \omega}\right)^{-1}\left(1+O_{L R, \omega}\left(O_{R R, \omega}-k\right)^{-1}\right), \\
& \mathcal{Q}_{R}=\left(O_{R R, \omega}-k-O_{R L, \omega}\left(O_{L L, \omega}+k\right)^{-1} O_{L R, \omega}\right)^{-1}\left(1+O_{R L, \omega}\left(O_{L L, \omega}+k\right)^{-1}\right), \\
& \mathcal{P}_{L}=\left(1+\left(O_{R R, \omega}+k\right)^{-1} O_{R L, \omega}\right)\left(O_{L L, \omega}-k-O_{L R, \omega}\left(O_{R R, \omega}+k\right)^{-1} O_{R L, \omega}\right)^{-1}, \\
& \mathcal{P}_{R}=\left(1+\left(O_{L L, \omega}-k\right)^{-1} O_{L R, \omega}\right)\left(O_{R R, \omega}+k-O_{R L, \omega}\left(O_{L L, \omega}-k\right)^{-1} O_{L R, \omega}\right)^{-1} .
\end{aligned}
$$

Inverting these relations it is then possible to express the gauge field as

$$
A_{-}=\frac{1}{2}\left[j_{-}^{L}+j_{-}^{R}-\left(\mathcal{Q}_{L}-\mathcal{Q}_{R}\right) J_{-}^{L}\right], \quad A_{+}=\frac{1}{2}\left[j_{+}^{L}+j_{+}^{R}-\left(\mathcal{P}_{L}^{t}-\mathcal{P}_{R}^{t}\right) J_{+}^{L}\right] .
$$

and the currents $J_{ \pm}^{L}$ as

$$
J_{-}^{L}=\left(\mathcal{Q}_{L}+\mathcal{Q}_{R}\right)^{-1} j_{-}, \quad J_{+}^{L}=\left(\mathcal{P}_{L}^{t}+\mathcal{P}_{R}^{t}\right)^{-1} j_{+},
$$

where

$$
j_{ \pm}=j_{ \pm}^{L}-j_{ \pm}^{R} .
$$

These results enable us to rewrite the first term in the Lagrangian for the action (3.14) as

$$
\begin{aligned}
-\sum_{a, b=L, R} \operatorname{tr} & {\left[\left(j_{+}^{a}-A_{+}\right) O_{a b, \omega}\left(j_{-}^{b}-A_{-}\right)\right] } \\
& =-\frac{1}{2} \operatorname{tr}\left[j_{+}\left(\mathcal{Q}_{L}+\mathcal{Q}_{R}\right)^{-1} j_{-}+j_{+}\left(\mathcal{P}_{L}+\mathcal{P}_{R}\right)^{-1} j_{-}\right] .
\end{aligned}
$$


The last term of (3.14) is proportional to the gauge field. We can therefore use the relation (3.30) to obtain

$$
\begin{aligned}
-k \operatorname{tr}\left[A_{-}\left(j_{+}^{L}-j_{+}^{R}\right)-A_{+}\left(j_{-}^{L}-j_{-}^{R}\right)\right]= & -k \operatorname{tr}\left[j_{+}^{L} j_{-}^{R}-j_{+}^{R} j_{-}^{L}\right] \\
& +\frac{1}{2} k \operatorname{tr}\left[j_{+}\left(\mathcal{Q}_{L}-\mathcal{Q}_{R}\right)\left(\mathcal{Q}_{L}+\mathcal{Q}_{R}\right)^{-1} j_{-}\right] \\
& -\frac{1}{2} k \operatorname{tr}\left[j_{+}\left(\mathcal{P}_{L}+\mathcal{P}_{R}\right)^{-1}\left(\mathcal{P}_{L}-\mathcal{P}_{R}\right) j_{-}\right] .
\end{aligned}
$$

The first term in (3.34) may be combined with the WZ terms associated with $g_{L}$ and $g_{R}$ using the Polyakov-Wiegmann formula [31]

$$
S_{\mathrm{WZ}, k}\left[g_{L}\right]-S_{\mathrm{WZ}, k}\left[g_{R}\right]-k \int d^{2} \sigma \operatorname{tr}\left[j_{+}^{L} j_{-}^{R}-j_{+}^{R} j_{-}^{L}\right]=S_{\mathrm{WZ}, k}\left[g_{L} g_{R}^{-1}\right] .
$$

Summing all these contributions gives

$$
\begin{aligned}
S_{\omega}\left[g=g_{L} g_{R}^{-1}\right]=S_{\mathrm{WZ}, k}[g]-\frac{1}{2} \int d^{2} \sigma \operatorname{tr}[ & j_{+}\left(1-k\left(\mathcal{Q}_{L}-\mathcal{Q}_{R}\right)\right)\left(\mathcal{Q}_{L}+\mathcal{Q}_{R}\right)^{-1} j_{-} \\
& \left.+j_{+}\left(\mathcal{P}_{L}+\mathcal{P}_{R}\right)^{-1}\left(1+k\left(\mathcal{P}_{L}-\mathcal{P}_{R}\right)\right) j_{-}\right],
\end{aligned}
$$

where the operators $\mathcal{Q}_{L}, \mathcal{Q}_{R}, \mathcal{P}_{L}$ and $\mathcal{P}_{R}$ are defined in (3.29). It is straightforward to check that, as indicated, this action only depends on $g_{L}$ and $g_{R}$ through the combination $g=g_{L} g_{R}^{-1}$. This is expected as a consequence of the $G_{\text {diag }}$ gauge symmetry (2.7).

\section{Equivalence with the Lukyanov model for $G=\mathrm{SU}(2)$}

In this section we prove that the action (3.14) corresponds to the Lukyanov model [1] for $G=\mathrm{SU}(2)$. Let us start by noting that $\mathrm{SU}(2)$ has rank one. Therefore in this case the operator $\omega$, introduced in section 3 , contains just a single parameter. In a slight abuse of notation we will also call this parameter $\omega$, with the operator given by multiplying by the identity (acting on the Cartan subalgebra). For $G=\mathrm{SU}(2)$, the action (3.14) thus defines a four-parameter integrable deformation of the SU(2) PCM. As a first order check of equivalence we observe that this is the same number of deformation parameters as in the Lukyanov model.

To demonstrate the full equivalence we shall start with the action (3.36), obtained after eliminating the gauge field. Partial identification of this four-parameter deformation with the Lukyanov model, that is to say with some deformation parameters set to zero, has already been shown in [2]. For this reason we use the same parameterisation of $g \in \mathrm{SU}(2)$ as in [2]. We then compute the corresponding metric and $B$-field and show that there exists a coordinate transformation, and a map between the parameters $\mathcal{A}_{L}, \mathcal{A}_{R}, k, \omega$ and Lukyanov's parameters $\kappa, p, h$ and $\bar{h}$, such that this metric and $B$-field coincides with those of [1].

Let us take the $\mathrm{SU}(2)$ group element

$$
g(r, \phi, \psi)=\mathrm{e}^{-T^{3}(\phi+\psi)}\left(r \mathbb{1}-2 \sqrt{1-r^{2}} T^{1}\right) \mathrm{e}^{-T^{3}(\phi-\psi)} .
$$


Here $T^{i}$ are the generators of $\mathfrak{s u}(2)$ satisfying

$$
\left[T^{i}, T^{j}\right]=\epsilon^{i j}{ }_{k} T^{k}, \quad \operatorname{tr}\left(T^{i} T^{j}\right)=-\frac{1}{2} \delta^{i j}, \quad i, j, k=1,2,3,
$$

where the totally anti-symmetric tensor $\epsilon^{i j k}$ is normalised as $\epsilon^{123}=+1$ and the $\mathfrak{s u}(2)$ indices are raised and lowered by $\delta^{i j}$ and its inverse. The $R$-matrix acts on the generators as

$$
R\left(T^{+}\right)=-i T^{+}, \quad R\left(T^{-}\right)=i T^{-}, \quad R\left(T^{3}\right)=0,
$$

where $T^{ \pm}=\frac{1}{\sqrt{2}}\left(T^{1} \pm i T^{2}\right)$.

The computation of the metric and $B$-field is rather lengthy but ultimately straightforward. In order to see the equivalence with the metric and $B$-field of Lukyanov's model one needs to perform the following coordinate transformations for the angle variables $\phi$ and $\psi$

$$
\begin{aligned}
& \phi=\chi_{1}+\frac{f_{L}^{+}+f_{R}^{+}}{4(1-k \omega)\left(1+\eta_{L}^{2}\right)\left(1+\eta_{R}^{2}\right) \mathcal{A}_{L} \mathcal{A}_{R}} \log \left[\frac{4+\left(\mathcal{A}_{L}-\mathcal{A}_{R}\right)^{2}}{4+\left(\mathcal{A}_{L}-\mathcal{A}_{R}\right)^{2}+4 r^{2} \mathcal{A}_{L} \mathcal{A}_{R}}\right], \\
& \psi=-\chi_{2}-\frac{f_{L}^{-}-f_{R}^{-}}{4(1+k \omega)\left(1+\eta_{L}^{2}\right)\left(1+\eta_{R}^{2}\right) \mathcal{A}_{L} \mathcal{A}_{R}} \log \left[\frac{4+\left(\mathcal{A}_{L}-\mathcal{A}_{R}\right)^{2}}{4+\left(\mathcal{A}_{L}-\mathcal{A}_{R}\right)^{2}+4 r^{2} \mathcal{A}_{L} \mathcal{A}_{R}}\right],
\end{aligned}
$$

where $f_{L, R}^{ \pm}$are given by

$$
\begin{aligned}
& f_{L}^{ \pm}=\mathcal{A}_{L}\left(1+\eta_{L}^{2}\right)\left(k \eta_{R}^{2} \pm\left(1+\eta_{R}^{2}\right)\left(1+\eta_{R}^{2}-k^{2}\right) \omega\right), \\
& f_{R}^{ \pm}=\mathcal{A}_{R}\left(1+\eta_{R}^{2}\right)\left(k \eta_{L}^{2} \pm\left(1+\eta_{L}^{2}\right)\left(1+\eta_{L}^{2}-k^{2}\right) \omega\right) .
\end{aligned}
$$

The resulting metric becomes block diagonal, i.e. $g_{r \chi_{1}}=g_{r \chi_{2}}=0$. We also introduce a new radial coordinate $z$ related to $r$ by

$$
r=\sqrt{\frac{(1-\kappa)(1+z)}{2(1-\kappa z)}},
$$

and define Lukyanov's parameters $(\kappa, p, h, \bar{h})$ as

$$
\begin{aligned}
\kappa & =\frac{\sqrt{4+\left(\mathcal{A}_{L}+\mathcal{A}_{R}\right)^{2}}-\sqrt{4+\left(\mathcal{A}_{L}-\mathcal{A}_{R}\right)^{2}}}{\sqrt{4+\left(\mathcal{A}_{L}+\mathcal{A}_{R}\right)^{2}}+\sqrt{4+\left(\mathcal{A}_{L}-\mathcal{A}_{R}\right)^{2}}}, \quad p^{2}=-\frac{\eta_{L}^{2}\left(1+\eta_{R}^{2}\right) \mathcal{A}_{R}}{\eta_{R}^{2}\left(1+\eta_{L}^{2}\right) \mathcal{A}_{L}}, \\
h_{ \pm} & =h \pm \bar{h}=-\frac{4 H_{ \pm}}{\left(\sqrt{4+\left(\mathcal{A}_{L}+\mathcal{A}_{R}\right)^{2}}+\sqrt{4+\left(\mathcal{A}_{L}-\mathcal{A}_{R}\right)^{2}}\right) H_{0}},
\end{aligned}
$$

where the quantities $H_{0}, H_{+}$and $H_{-}$are given by

$$
\begin{aligned}
& H_{0}=\sqrt{\left(1+\eta_{L}^{2}\right)\left(1+\eta_{R}^{2}\right)}\left(1-k^{2} \omega^{2}\right), \\
& H_{+}=k \sqrt{\eta_{L}^{2} \eta_{R}^{2}+\omega^{2}\left(1+\eta_{L}^{2}\right)\left(1+\eta_{R}^{2}\right)\left(4+\mathcal{A}_{L}^{2}+\mathcal{A}_{R}^{2}+\omega^{2}\left(1+\eta_{L}^{2}-k^{2}\right)\left(1+\eta_{R}^{2}-k^{2}\right)\right)}, \\
& H_{-}=\omega\left(k^{2}+\left(1+\eta_{L}^{2}\right)\left(1+\eta_{R}^{2}\right)\right) .
\end{aligned}
$$

With these identifications we indeed recover the metric and $B$-field of [1]. In particular, up to a total derivative, the Lagrangian corresponding to $(3.36)$ is $[1,2]$

$$
\begin{aligned}
L=T & {\left[U(z) \partial_{+} z \partial_{-} z+D(z) \partial_{+} \chi_{1} \partial_{-} \chi_{1}+\hat{D}(z) \partial_{+} \chi_{2} \partial_{-} \chi_{2}\right.} \\
& \left.+[C(z)+B(z)] \partial_{+} \chi_{1} \partial_{-} \chi_{2}+[C(z)-B(z)] \partial_{+} \chi_{2} \partial_{-} \chi_{1}\right]
\end{aligned}
$$


where we have rewritten the Lukyanov background using new angle variables $\left(\chi_{1}, \chi_{2}\right)$, related to the original ones $(v, w)$ through $\chi_{1}=\frac{1}{2} R^{-1}(v-w), \chi_{2}=\frac{1}{2}(v+w)[2]$. The overall factor $T$ is equal to

$$
T=\frac{2\left(\left(1+\eta_{L}^{2}\right)\left(1+\eta_{R}^{2}\right)+k^{2}\right)}{2+\eta_{L}^{2}+\eta_{R}^{2}}
$$

while the components of (4.9) are

$$
\begin{aligned}
& U(z)=\frac{m^{2}}{4\left(1-z^{2}\right)\left(1-\kappa^{2} z^{2}\right)} \\
& D(z)=R^{2}(1+z)\left[2+\kappa\left(p^{2}+p^{-2}\right)-\kappa\left(2 \kappa+p^{2}+p^{-2}\right) z\right] Q(z), \\
& \hat{D}(z)=(1-z)\left[2+\kappa\left(p^{2}+p^{-2}\right)+\kappa\left(2 \kappa+p^{2}+p^{-2}\right) z\right] Q(z), \\
& C(z)=\kappa\left(p^{2}-p^{-2}\right) R\left(1-z^{2}\right) Q(z) \\
& B(z)=-\frac{2 R m}{c+\bar{c}}\left[h\left(c^{2}-1\right)(\bar{c}-z)-\bar{h}\left(\bar{c}^{2}-1\right)(c+z)\right] Q(z),
\end{aligned}
$$

where $Q(z)$ is given by

$$
Q(z)=\frac{(c+1)(\bar{c}-1)}{4\left(1-\kappa^{2}\right)(c+z)(\bar{c}-z)} .
$$

Finally, we recall the definitions of $c, \bar{c}, m$ and $R$,

$$
\begin{aligned}
c & =\sqrt{\frac{1+h^{2}}{\kappa^{2}+h^{2}}}, & \bar{c} & =\sqrt{\frac{1+\bar{h}^{2}}{\kappa^{2}+\bar{h}^{2}}}, \\
m & =\sqrt{\left(\kappa+p^{2}\right)\left(\kappa+p^{-2}\right)}, & R & =\sqrt{\frac{(c-1)(\bar{c}+1)}{(c+1)(\bar{c}-1)}} .
\end{aligned}
$$

The expressions of $c, \bar{c}$ and $m$ in terms of the parameters $\mathcal{A}_{L}, \mathcal{A}_{R}, k$ and $\omega$ are cumbersome. We shall therefore not reproduce them here. However, let us point out that the relations (4.7) and (4.13) lead to a simple expression for $R$,

$$
R=\frac{1-k \omega}{1+k \omega}
$$

This expression is interesting because it has simple limits. Indeed, we have $R=1$ when $\omega=0$ or $k=0$. This result is consistent with those obtained previously in [2].

\section{Conclusion}

In this paper we have presented a new multi-parameter integrable deformation of the PCM for a general group $G$. The first step of its construction was the derivation of the integrable bi-Yang-Baxter deformation of the PCM plus WZ term. The second was the implementation of a general TsT transformation mixing the Cartan generators of the two copies of $G$. 
This multi-parameter integrable model generalises Lukyanov's four-parameter deformation of the SU(2) PCM [1] to arbitrary group $G$. Therefore the construction confirms the proposal of [2] on the algebraic origin of the four parameters: two correspond to the bi-Yang-Baxter deformation, one parameterises the coupling to the WZ term, and the final parameter is generated by a TsT transformation.

There are a number of possible open questions whose investigation would further probe the properties of this integrable $\sigma$-model. One of the most important is the study of its classical Poisson structure, Hamiltonian integrability and twist function in the spirit of $[10,11,13,32]$. To determine the twist function, it is enough to consider the threeparameter case. Indeed, the twist function is not changed under a TsT transformation [32]. One particular aim is to understand the $q$-deformed algebra of hidden charges. Furthermore, the extension to the affine algebra as considered in [33] for the Yang-Baxter $\sigma$-model would be interesting to investigate (see also $[34,35,16]$ for the $\mathrm{SU}(2)$ case). Finally, studying this $\sigma$-model at the Hamiltonian level would indicate if it is also possible to reinterpret it as a dihedral affine Gaudin model [36].

In [37] the Yang-Baxter deformation of the PCM plus WZ term of [14] was recast in the framework of $\mathcal{E}$-models [38, 39] (a first-order action defined on the Drinfel'd double). Understanding how to formulate the bi-Yang-Baxter deformation of the PCM plus WZ term (and TsT transformations thereof) presented in sections 2 (and 3) in this language may prove useful in gaining a deeper understanding of the underlying algebraic structure of the model.

Setting $\eta_{L}=\eta_{R}=\omega=0$ and $k=1$, the deformed action simplifies to the WZW action for the group $G$. This model is conformal, as are its deformations associated with TsT transformations. It would be interesting to investigate which other points in parameter space correspond to conformal sigma models at the quantum level and hence define string backgrounds. In particular, this would involve generalising the one-loop renormalisation analysis, including UV and IR fixed points, of [1] beyond the SU(2) case.

Finally, there are a class of superstring backgrounds for which the Green-Schwarz worldsheet action takes the form (at least in part) of an integrable supercoset $\sigma$-model [4044]. For the maximally symmetric $\mathrm{AdS}_{5} \times S^{5}$ background the $\mathrm{PSU}(2,2 \mid 4) /(\mathrm{SO}(1,4) \times \mathrm{SO}(5))$ supercoset model of [40] captures the full Green-Schwarz string. Generalising the bosonic construction of [10], the Yang-Baxter deformation of this model was constructed in [45, 46].

Particularly relevant to the constructions of this paper are string backgrounds for which the superisometry takes the form of a product group. For example, the $\operatorname{AdS}_{3} \times S^{3} \times T^{4}$ background is related to the supercoset $\mathrm{PSU}(1,1 \mid 2)^{2} /(\mathrm{SU}(1,1) \times \mathrm{SU}(2))_{\text {diag }}$, and the $\mathrm{AdS}_{3} \times$ $S^{3} \times S^{3} \times S^{1}$ background to the supercoset $D(2,1 ; \alpha)^{2} /(\mathrm{SU}(1,1) \times \mathrm{SU}(2) \times \mathrm{SU}(2))_{\operatorname{diag}}[47] . \mathrm{In}$ these cases one can construct a bi-Yang-Baxter deformation of the supercoset $\sigma$-model [25], or alternatively introduce a WZ term [48]. The results presented in this paper provide the first steps towards combining these two constructions into a single three-parameter model, on top of which further parameters may be introduced via TsT transformations. 


\section{Acknowledgments}

We thank S. Lacroix and B. Vicedo for comments on the draft. The work of FD, TK and $\mathrm{MM}$ is partially supported by the French Agence Nationale de la Recherche (ANR) under grant ANR-15-CE31-0006 DefIS. The work of BH is partially supported by grant no. 615203 from the European Research Council under the FP7.

Open Access. This article is distributed under the terms of the Creative Commons Attribution License (CC-BY 4.0), which permits any use, distribution and reproduction in any medium, provided the original author(s) and source are credited.

\section{References}

[1] S.L. Lukyanov, The integrable harmonic map problem versus Ricci flow, Nucl. Phys. B $\mathbf{8 6 5}$ (2012) 308 [arXiv: 1205.3201] [INSPIRE].

[2] B. Hoare, R. Roiban and A.A. Tseytlin, On deformations of $A d S_{n} \times S^{n}$ supercosets, JHEP 06 (2014) 002 [arXiv: 1403.5517] [inSPIRE].

[3] V.A. Fateev, The $\sigma$-model (dual) representation for a two-parameter family of integrable quantum field theories, Nucl. Phys. B 473 (1996) 509 [INSPIRE].

[4] E. Witten, Nonabelian Bosonization in Two-Dimensions, Commun. Math. Phys. 92 (1984) 455 [INSPIRE].

[5] J.H. Horne and G.T. Horowitz, Exact black string solutions in three-dimensions, Nucl. Phys. B 368 (1992) 444 [hep-th/9108001] [INSPIRE].

[6] A. Giveon and M. Roček, Generalized duality in curved string backgrounds, Nucl. Phys. B 380 (1992) 128 [hep-th/9112070] [INSPIRE].

[7] C. Klimčík, Yang-Baxter $\sigma$-models and dS/AdS T duality, JHEP 12 (2002) 051 [hep-th/0210095] [INSPIRE].

[8] C. Klimčík, On integrability of the Yang-Baxter $\sigma$-model, J. Math. Phys. 50 (2009) 043508 [arXiv:0802.3518] [INSPIRE].

[9] C. Klimčík, Integrability of the bi-Yang-Baxter $\sigma$-model, Lett. Math. Phys. 104 (2014) 1095 [arXiv: 1402.2105] [INSPIRE].

[10] F. Delduc, M. Magro and B. Vicedo, On classical q-deformations of integrable $\sigma$-models, JHEP 11 (2013) 192 [arXiv: 1308.3581] [INSPIRE].

[11] F. Delduc, S. Lacroix, M. Magro and B. Vicedo, On the Hamiltonian integrability of the bi-Yang-Baxter $\sigma$-model, JHEP 03 (2016) 104 [arXiv:1512.02462] [INSPIRE].

[12] I. Kawaguchi and K. Yoshida, Hybrid classical integrability in squashed $\sigma$-models, Phys. Lett. B 705 (2011) 251 [arXiv:1107.3662] [INSPIRE].

[13] F. Delduc, S. Lacroix, M. Magro and B. Vicedo, On q-deformed symmetries as Poisson-Lie symmetries and application to Yang-Baxter type models, J. Phys. A 49 (2016) 415402 [arXiv: 1606.01712] [INSPIRE].

[14] F. Delduc, M. Magro and B. Vicedo, Integrable double deformation of the principal chiral model, Nucl. Phys. B 891 (2015) 312 [arXiv:1410.8066] [INSPIRE]. 
[15] I. Kawaguchi, D. Orlando and K. Yoshida, Yangian symmetry in deformed WZNW models on squashed spheres, Phys. Lett. B 701 (2011) 475 [arXiv:1104.0738] [INSPIRE].

[16] I. Kawaguchi and K. Yoshida, A deformation of quantum affine algebra in squashed Wess-Zumino-Novikov-Witten models, J. Math. Phys. 55 (2014) 062302 [arXiv:1311.4696] [INSPIRE].

[17] I. Kawaguchi, T. Matsumoto and K. Yoshida, Jordanian deformations of the $A d S_{5} \times S^{5}$ superstring, JHEP 04 (2014) 153 [arXiv:1401.4855] [INSPIRE].

[18] T. Matsumoto and K. Yoshida, Lunin-Maldacena backgrounds from the classical Yang-Baxter equation - Towards the gravity/CYBE correspondence, JHEP 06 (2014) 135 [arXiv: 1404.1838] [INSPIRE].

[19] T. Matsumoto and K. Yoshida, Schrödinger geometries arising from Yang-Baxter deformations, JHEP 04 (2015) 180 [arXiv:1502.00740] [INSPIRE].

[20] T. Matsumoto and K. Yoshida, Integrability of classical strings dual for noncommutative gauge theories, JHEP 06 (2014) 163 [arXiv:1404.3657] [INSPIRE].

[21] T. Matsumoto and K. Yoshida, Yang-Baxter $\sigma$-models based on the CYBE, Nucl. Phys. B 893 (2015) 287 [arXiv:1501.03665] [INSPIRE].

[22] S.J. van Tongeren, On classical Yang-Baxter based deformations of the AdS $S_{5} \times S^{5}$ superstring, JHEP 06 (2015) 048 [arXiv: 1504.05516] [INSPIRE].

[23] S.J. van Tongeren, Yang-Baxter deformations, AdS/CFT and twist-noncommutative gauge theory, Nucl. Phys. B 904 (2016) 148 [arXiv:1506.01023] [InSPIRE].

[24] D. Osten and S.J. van Tongeren, Abelian Yang-Baxter deformations and TsT transformations, Nucl. Phys. B 915 (2017) 184 [arXiv:1608.08504] [INSPIRE].

[25] B. Hoare, Towards a two-parameter q-deformation of $A d S_{3} \times S^{3} \times M^{4}$ superstrings, Nucl. Phys. B 891 (2015) 259 [arXiv:1411.1266] [INSPIRE].

[26] C. Klimčík and A.A. Tseytlin, Duality invariant class of exact string backgrounds, Phys. Lett. B 323 (1994) 305 [hep-th/9311012] [INSPIRE].

[27] S.A. Frolov, R. Roiban and A.A. Tseytlin, Gauge-string duality for superconformal deformations of $N=4$ super Yang-Mills theory, JHEP 07 (2005) 045 [hep-th/0503192] [INSPIRE].

[28] S. Frolov, Lax pair for strings in Lunin-Maldacena background, JHEP 05 (2005) 069 [hep-th/0503201] [INSPIRE].

[29] L.F. Alday, G. Arutyunov and S. Frolov, Green-Schwarz strings in TsT-transformed backgrounds, JHEP 06 (2006) 018 [hep-th/0512253] [INSPIRE].

[30] D. Bykov, Complex structure-induced deformations of $\sigma$-models, JHEP 03 (2017) 130 [arXiv: 1611.07116] [INSPIRE].

[31] A.M. Polyakov and P.B. Wiegmann, Theory of Nonabelian Goldstone Bosons, Phys. Lett. B 131 (1983) 121 [INSPIRE].

[32] B. Vicedo, Deformed integrable $\sigma$-models, classical R-matrices and classical exchange algebra on Drinfel'd doubles, J. Phys. A 48 (2015) 355203 [arXiv:1504.06303] [INSPIRE].

[33] F. Delduc, T. Kameyama, M. Magro and B. Vicedo, Affine q-deformed symmetry and the classical Yang-Baxter $\sigma$-model, JHEP 03 (2017) 126 [arXiv:1701. 03691] [INSPIRE]. 
[34] I. Kawaguchi, T. Matsumoto and K. Yoshida, The classical origin of quantum affine algebra in squashed $\sigma$-models, JHEP 04 (2012) 115 [arXiv:1201.3058] [INSPIRE].

[35] I. Kawaguchi, T. Matsumoto and K. Yoshida, On the classical equivalence of monodromy matrices in squashed $\sigma$-model, JHEP 06 (2012) 082 [arXiv:1203.3400] [INSPIRE].

[36] B. Vicedo, On integrable field theories as dihedral affine Gaudin models, arXiv:1701.04856 [INSPIRE].

[37] C. Klimčík, Yang-Baxter $\sigma$-model with WZNW term as $\mathcal{E}$-model, Phys. Lett. B $772(2017)$ 725 [arXiv: 1706. 08912] [INSPIRE].

[38] C. Klimčík, $\eta$ and $\lambda$ deformations as $\mathcal{E}$-models, Nucl. Phys. B 900 (2015) 259 [arXiv: 1508.05832] [INSPIRE].

[39] C. Klimčík, Poisson-Lie T-duals of the bi-Yang-Baxter models, Phys. Lett. B 760 (2016) 345 [arXiv: 1606.03016] [INSPIRE].

[40] R.R. Metsaev and A.A. Tseytlin, Type IIB superstring action in $A d S_{5} \times S^{5}$ background, Nucl. Phys. B 533 (1998) 109 [hep-th/9805028] [INSPIRE].

[41] N. Berkovits, M. Bershadsky, T. Hauer, S. Zhukov and B. Zwiebach, Superstring theory on $A d S_{2} \times S^{2}$ as a coset supermanifold, Nucl. Phys. B 567 (2000) 61 [hep-th/9907200] [INSPIRE].

[42] I. Bena, J. Polchinski and R. Roiban, Hidden symmetries of the $A d S_{5} \times S^{5}$ superstring, Phys. Rev. D 69 (2004) 046002 [hep-th/0305116] [INSPIRE].

[43] K. Zarembo, Strings on Semisymmetric Superspaces, JHEP 05 (2010) 002 [arXiv: 1003.0465] [INSPIRE].

[44] L. Wulff, On integrability of strings on symmetric spaces, JHEP 09 (2015) 115 [arXiv: 1505.03525] [INSPIRE].

[45] F. Delduc, M. Magro and B. Vicedo, An integrable deformation of the $A d S_{5} \times S^{5}$ superstring action, Phys. Rev. Lett. 112 (2014) 051601 [arXiv: 1309.5850] [INSPIRE].

[46] F. Delduc, M. Magro and B. Vicedo, Derivation of the action and symmetries of the q-deformed $A d S_{5} \times S^{5}$ superstring, JHEP 10 (2014) 132 [arXiv:1406.6286] [INSPIRE].

[47] A. Babichenko, B. Stefanski Jr. and K. Zarembo, Integrability and the $A d S_{3} / C F T_{2}$ correspondence, JHEP 03 (2010) 058 [arXiv: 0912.1723] [INSPIRE].

[48] A. Cagnazzo and K. Zarembo, B-field in $A d S_{3} / C F T_{2}$ Correspondence and Integrability, JHEP 11 (2012) 133 [Erratum ibid. 04 (2013) 003] [arXiv: 1209.4049] [INSPIRE]. 